\title{
Halo coronal mass ejections and geomagnetic storms
}

\author{
Nat Gopalswamy \\ NASA Goddard Space Flight Center, Greenbelt, Maryland, U.S.A.
}

(Received June 21, 2008; Revised October 27, 2008; Accepted October 27, 2008; Online published May 29, 2009)

\begin{abstract}
In this letter, I show that the discrepancies in the geoeffectiveness of halo coronal mass ejections (CMEs) reported in the literature arise due to the varied definitions of halo CMEs used by different authors. In particular, I show that the low geoeffectiveness rate is a direct consequence of including partial halo CMEs. The geoeffectiveness of partial halo CMEs is lower because they are of low speed and likely to make a glancing impact on Earth.
\end{abstract}

Key words: Coronal mass ejections, geomagnetic storms, geoeffectiveness, halo CMEs.

\section{Introduction}

Coronal mass ejections (CMEs) that appear to surround the occulting disk of the observing coronagraphs in skyplane projection are known as halo CMEs (Howard et al., 1982). Halo CMEs are fast and wide on the average and are associated with flares of greater X-ray importance because only energetic CMEs expand rapidly to appear above the occulting disk early in the event (Gopalswamy et al., 2007). Extensive observations from the Solar and Heliospheric Observatory (SOHO) mission's Large Angle and Spectrometric Coronagraphs (LASCO) have shown that full halos constitute $\sim 3.6 \%$ of all CMEs, while CMEs with width $\geq 120^{\circ}$ account for $\sim 11 \%$ (Gopalswamy, 2004). Full halos have an apparent width $(W)$ of $360^{\circ}$, while partial halos have $120^{\circ} \leq W<360^{\circ}$. Halo CMEs are said to be frontsided if the site of eruption (also known as the solar source) can be identified on the visible disk usually identified as the location of $\mathrm{H}$-alpha flares or filament eruptions. Details on how to identify the solar sources can be found in Gopalswamy et al. (2007). Halos with their sources within $\pm 45^{\circ}$ of the central meridian are known as disk halos, while those with a central meridian distance (CMD) beyond $\pm 45^{\circ}$ but not beyond $\pm 90^{\circ}$ are known as limb halos. Disk halos are likely to arrive at Earth and cause geomagnetic storms, while limb halos only impact Earth with their flanks and hence are less geoeffective (see Gopalswamy et al., 2007).

Since CMEs propagate approximately radially from the Sun (except for a small eastward deflection due to solar rotation-see Gosling et al., 1987), disk halos are likely hit Earth. Of course, the interplanetary counterpart of CMEs (ICMEs) must contain southward magnetic field component $\left(B_{s}\right)$ to be geoeffective. It is well known that the intensity of the resulting magnetic storm depends on the magnitude of $B_{s}$ and the speed $V$ with which the CME impacts Earth's magnetosphere (see e.g., Gonzalez et al., 1994; Tsurutani and Gonzalez, 1997). Halo CMEs are more energetic (av-

Copyright (c) The Society of Geomagnetism and Earth, Planetary and Space Sciences (SGEPSS); The Seismological Society of Japan; The Volcanological Society of Japan; The Geodetic Society of Japan; The Japanese Society for Planetary Sciences; TERRAPUB. No claim is made to original U.S. Government works. erage speed is $\sim 1000 \mathrm{~km} / \mathrm{s}$ compared to $\sim 470 \mathrm{~km} / \mathrm{s}$ for ordinary CMEs), so the $V$ part is expected to be high. When CMEs are aimed directly at Earth, the ICMEs are likely to arrive at Earth as magnetic clouds (MCs), which are a subset of ICMEs that have flux rope structure. Since Earth passes through the nose of the $\mathrm{MC}$, the chance of encountering $B_{S}$ somewhere within the MC is high (except for unipolar MCs with north-pointing axis—see Yurchyshyn et al., 2001). When the ICME is shock-driving, the sheath portion lying between the shock and the MC may also play a significant role in producing geomagnetic storms (see e.g., Gosling et al., 1990). The sheath may have intervals of north and south-pointing magnetic fields, in addition to the varying field orientation in the MC portion. An Earth-directed halo CME leads to a situation whereby Earth passes through the nose of the shock, where the sheath field is most intense and may cause intense magnetic storm if south-pointing. Thus the ability of a halo CME in producing a geomagnetic storm depends on the structure of its interplanetary counterpart (the ICME event).

Since halos became common place in the SOHO era, there have been several attempts to characterize their geoeffectiveness (see e.g., Zhao and Webb, 2003; Yermolaev and Yermolaev, 2003; Kim et al., 2005; Yermolaev et al., 2005; Gopalswamy et al., 2007). Using CMEs from the rise phase of solar cycle 23, St. Cyr et al. (2000) concluded that $\sim 75 \%$ of the frontside CMEs are geoeffective. In most of the works discussed here, the geoeffectiveness is defined as the ability of a CME or an interplanetary structure to cause geomagnetic storms with intensity $\left(D_{\text {st }}\right) \leq-50 \mathrm{nT}$. While most of the intense storms are caused by CMEs, moderate storms $\left(-100 \mathrm{nT}<D_{\text {st }}<-50 \mathrm{nT}\right)$ may also be caused by other structures such as corotating interaction regions. Zhao and Webb (2003) found an overall geoeffectiveness rate of $\sim 64 \%$ for frontside halos detected up to the solar maximum in 2000. Yermolaev and Yermolaev (2003) used data from the period 1976-2000 and came up with a lower rate of $40-50 \%$. Michalek et al. (2006) found that $\sim 56 \%$ of frontside halos are geoeffective, but they did not use all the halos because of limitations in the method of obtaining 
space speeds. Kim et al. (2005) reported that only about $40 \%$ of the frontside halos are geoeffective. Yermolaev et al. (2005) compiled published results and noted that that the geoeffectiveness rate varied from $<40 \%$ to $>80 \%$. Recently Gopalswamy et al. (2007) analyzed 378 halo CMEs covering almost whole of solar cycle 23 and found that $\sim 71 \%$ of frontside halos are geoeffective. Zhao and Webb (2003) and Gopalswamy et al. (2007) used the same definition of halo CMEs $\left(W=360^{\circ}\right)$ and obtained similar geoeffectiveness rates. On the other hand, Kim et al. (2005) and Yermolaev and Yermolaev (2003) defined their halos as CMEs with $W \geq 120^{\circ}$ and obtained the lower geoeffectiveness rate. The purpose of this letter is to show that the discrepancy in the rate of geoeffectiveness can simply be explained by the different definition of halo CMEs used by these authors. We take Kim et al. (2005) and Gopalswamy et al. (2007) representing the low and high geoeffectiveness rates, respectively. Both these works have published their list of events and the study periods have maximum overlap. They also use data from the same instrument (SOHO/LASCO), so the comparison is straightforward. Finally, both works use the same definition of geoeffectiveness: the ability of a CME to produce a geomagnetic storm with $D_{\text {st }} \leq-50 \mathrm{nT}$.

\section{Geoeffectiveness of Partial Halo CMEs}

Kim et al. (2005) investigated 305 CMEs (1997 to 2003) that included full $\left(W=360^{\circ}\right)$ and partial halos $\left(120^{\circ}<\right.$ $W<360^{\circ}$ ) and found that 121 of them were geoeffective. On the other hand Gopalswamy et al. (2007) studied 378 full halos for the period 1996 to 2005 . For making a proper comparison, we first separate the full and partial halos in Kim et al. (2005). We then use the geoeffectiveness of a subset of full halos from Gopalswamy et al. (2007) corresponding to the Kim et al. (2005) study period to estimate the geoeffectiveness of partial halo CMEs.

Among the 378 full halos reported by Gopalswamy et al. (2007), 168 occurred during the period 1997 to 2003 (the study period of Kim et al., 2005). Many geomagnetic storms during the study period had multiple CME association, so we eliminate 68 such CMEs in estimating the geoeffectiveness rate. Sixty five of the remaining 100 frontside full halos $(65 \%)$ were geoeffective. Note that this rate is close to the one obtained by Zhao and Webb (2003). Excluding the full halos from the CMEs in Kim et al. (2005), we get 205 partial halos (305 minus 100), out of which 56 (121 minus 65) were geoeffective. Thus we get a geoeffectiveness rate of $27 \%$ (56 out of 205) for the partial halos alone. The $40 \%$ geoeffectiveness rate obtained by Kim et al. (2005) is thus a consequence of combining highly geoeffective $(65 \%)$ full halos and marginally geoeffective $(27 \%)$ partial halos.

Note that we used the CME data for the period 19972003 in the above calculation. To extend this result to the whole study period (1996-2005) considered by Gopalswamy et al. (2007), we need to estimate the faction of partial halos that are geoeffective. Since Gopalswamy et al. (2007) did not include partial halos in their study, we estimate the geoeffectiveness of partial halos by extrapolation. To do this, we make use of the fact that $3.5 \%$ of all CMEs are full halos, while $11 \%$ are full + partial halos (Gopalswamy, 2004). Assuming that these fractions apply equally well to the front and backside CMEs and recalling that 229 full halos were frontsided, one can estimate the frontside wide CMEs as $(11 \% / 3.5 \%) \times 229=720$. Therefore, $720-229=491$ is the likely number of partial halos. At the $27 \%$ geoeffectiveness rate estimated above, 134 of the 491 partial halos are expected to be geoeffective. Therefore, out of the 720 wide CMEs, 163 full halos and 134 partial halos (total of 297 or $41 \%$ ) are geoeffective. This is virtually the same as the $40 \%$ rate obtained by Kim et al. (2005) and confirms that inclusion of partial halos lowers the overall geoeffectiveness rate. Note that the geoeffectiveness of halos varies with the phase of the solar cycle (see Gopalswamy et al., 2007, figure 8; Zhao and Webb, 2003) and the way multiple halos associated with a given storm are treated. We estimate that these effects can account for an additional $10 \%$ variation.

\subsection{Why are partial halos less geoeffective?}

In studying the geoeffectiveness of CMEs as a function of source latitude, Gopalswamy et al. (2007) found that the strongly geoeffective $\left(D_{\mathrm{st}} \leq-100 \mathrm{nT}\right)$ and moderately geoeffective $\left(-50 \geq D_{\text {st }}>-100 \mathrm{nT}\right)$ CMEs have average longitudes of $\mathrm{W} 10$ and E03, respectively. The non-geoeffective CMEs have an average longitude similar to the moderately geoeffective CMEs (E02). Furthermore, the fraction of limb halos steadily increases from $17 \%$ for strongly-geoeffective, to $31 \%$ for moderately geoeffective, and $37 \%$ for non-geoeffective CMEs. The average speeds also decrease in the same order (see figure 9 of Gopalswamy et al., 2007). From these observations, Gopalswamy et al. (2007) concluded that non-geoeffective CMEs are relatively slower, originate predominantly from the eastern hemisphere, and have a greater central meridian distance. Partial halos generally have properties similar to the moderately geoeffective and non-geoeffective halos. Thus partial halos are less energetic and do not expand enough to fully surround the occulting disk within the LASCO field of view.

Gopalswamy et al. (2007) also reported that the geoeffectiveness rates of limb and disk halos as $60 \%$ and $75 \%$, respectively. The geoeffectiveness of partial halos originating closer to the limb is expected to be even lower because they are slower and less likely to impact Earth. Thus, inclusion of partial halos reduces the overall geoeffectiveness rate of halo CMEs and hence correctly explains the lower geoeffectiveness rates reported in the literature.

\section{Discussion and Conclusions}

One of the important aspects of CME geoeffectiveness studies is to identify the solar source of CMEs. One might argue that the geoeffectiveness may be overestimated when frontsided CMEs are mistakenly classified as backsided CMEs. This can happen only when frontsided halos have no disk signature. There may also be cases in which a fortuitous disk activity coincides with a backside CME and hence a backside CME gets classified as a frontside CME. Sometimes, one observes a full halo, which may be a combination of multiple CMEs occurring at different position angles. Such occurrences are generally rare and cannot ac- 
count for the large discrepancies in the reported geoeffectiveness rate.

To clarify the identification of solar sources of halo CMEs, let us consider the association between CMEs and soft X-ray flares, one of the obvious indicators of disk activity. Whenever the eruption occurs on the frontside (CMD in the range 0 to $90^{\circ}$ ), we observe a soft X-ray flare. The halo CME appears asymmetric when the solar source has a larger CMD, typically beyond $45^{\circ}$. When the eruption is behind the limb, but not too far behind, we usually observe some EUV dimming above the concerned limb, but no soft X-ray flare is observed because the flare gets occulted by the solar limb. When a flare is partially occulted by a limb, the soft X-ray light curve tends to be very gradual and we observe the CME above the occulting limb. The extreme case is a backside CME whose associated flare is completely occulted, and we see no disk activity. In some of these cases one can see EUV dimming around most part of the solar disk, indicating a backsided eruption. This kind of relationship between the soft X-ray flare and CMEs can be easily seen by tracking a large active region (AR) during its disk passage and eventual disappearance behind the west limb (e.g., AR 10486 reported in Gopalswamy et al., 2005).

When one starts from geomagnetic storms and relate them to CMEs near the Sun, occasionally it becomes difficult to identify the CME. Zhang et al. (2007) were not able to identify wide CMEs or their solar sources for $\sim 10 \%$ of large geomagnetic storms. Zhang et al. (2007) started with large geomagnetic storms and searched for CMEs and their solar sources. The solar and geomagnetic events were separated by more than a day to a few days. On the other hand, Gopalswamy et al. (2007) started with halo CMEs and identified their solar sources. Observations of the halo $\mathrm{CME}$ and the associated disk signature are nearly simultaneous. Halo CMEs are more energetic (see figure 4 of Gopalswamy et al., 2007), so there is usually a prompt (and strong) disk signature if the halo is frontsided. Thus, the solar source identification for geomagnetic storms and that for halo CMEs do not have the same level of difficulty. Furthermore, geomagnetic storms can also be caused by nonhalo CMEs. If a CME originates close to the disk center and arrives at Earth with a southward magnetic field component, it will cause a geomagnetic storm. It is known that some magnetic clouds, which are interplanetary CMEs (ICMEs) with flux rope structure, are associated with nonhalo CMEs. A recent statistical study (Gopalswamy et al., 2008 ) finds that only $\sim 63 \%$ of magnetic clouds are associated with full halos. The fraction increases to $\sim 86 \%$ when full and partial halos are combined. The remaining $14 \%$ of magnetic clouds are associated with non-halo CMEs originating from close to the disk center. Since magnetic clouds constitute the most geoeffective subset of ICMEs, one expects that some magnetic clouds associated with non-halo CMEs are also geoeffective.

In conclusion, we confirm that the lower rate of geoeffectiveness obtained by some authors is due to the inclusion of partial halos. The reported variation in geoeffectiveness rates can be readily explained by the different definition of halo CMEs used by different authors. Partial halos are less energetic and generally originate far from the disk center, so most of them behave similar to the nongeoeffective CMEs.

Acknowledgments. Work supported by NASA's LWS and TR\&T programs.

\section{References}

Gonzalez, W. D., J. A. Joselyn, Y. Kamide, H. W. Kroehl, G. Rostoker, B. T. Tsurutani, and V. M. Vasyliunas, What is a geomagnetic storm?, $J$. Geophys. Res., 99, 5771, 1994.

Gopalswamy, N., A global picture of CMEs in the inner heliosphere, in The Sun and the Heliosphere as an Integrated System, edited by G. Poletto and S. T. Suess, chap. 8, p. 201, Kluwer Acad., Boston, 2004.

Gopalswamy, N., S. Yashiro, Y. Liu, G. Michalek, A. Vourlidas, M. L. Kaiser, and R. A. Howard, Coronal mass ejections and other extreme characteristics of the 2003 October-November solar eruptions, J. Geophys. Res., 110, A09S15, doi:10.1029/2004JA010958, 2005.

Gopalswamy, N., S. Yashiro, and S. Akiyama, Geoeffectiveness of halo coronal mass ejections, J. Geophys. Res., 112, A06112, doi: 10.1029/2006JA012149, 2007.

Gopalswamy, N., S. Akiyama, S. Yashiro, G. Michalek, and R. P. Lepping, Solar sources and geospace consequences of interplanetary magnetic clouds observed during solar cycle 23, J. Atm. Sol. Terr. Phys., 70, 245, doi:10.1016/j.jastp.2007.08.070, 2008.

Gosling, J. T., M. F. Thomsen, S. J. Bame, and R. D. Zwickl, The eastward deflection of fast coronal mass ejecta in interplanetary space, $J$. Geophys. Res., 92, 12399, 1987.

Gosling, J. T., D. J. McComas, J. L. Phillips, and S. J. Bame, Coronal mass ejections and large geomagnetic storms, Geophys. Res. Lett., 17, 901, 1990.

Howard, R. A., D. J. Michels, N. R. Sheeley, Jr., and M. J. Koomen, The observation of a coronal transient directed at earth, Astrophys. J., 263, L101, 1982.

Kim, R.-S., K.-S. Cho, Y.-J. Moon, Y.-H. Kim, Y. Yi, M. Dryer, S.-C. Bong, and Y.-D. Park, Forecast evaluation of the coronal mass ejection (CME) geoeffectiveness using halo CMEs from 1997 to 2003, $J$. Geophys. Res., 110, A11104, doi:10.1029/2005JA011218, 2005.

Michalek, G., N. Gopalswamy, A. Lara, and S. Yashiro, Properties and geoeffectiveness of halo CMEs, Space Weather, 4, S10003, doi:10.1029/2005SW000218, 2006.

St. Cyr, O. C. et al., Properties of coronal mass ejections: SOHO LASCO observations from January 1996 to June 1998, J. Geophys. Res., 105, $18,169,2000$.

Tsurutani, B. T. and W. D. Gonzalez, The interplanetary causes of magnetic storms: A review, in Magnetic Storms, edited by B. T. Tsurutani, W. D. Gonzalez, Y. Kamide, and J. K. Arballo, p. 77, AGU Monograph 98, AGU, Washington DC, 1997.

Yermolaev, Yu. I. and M. Yu. Yermolaev, Statistical relationships between solar, interplanetary, and geomagnetic disturbances, 1976-2000: 3, Cosmic Res., 41, 539, 2003.

Yermolaev, Yu. I., M. Yu. Yermolaev, G. N. Zastenker, L. M. Zelenyi, A. A. Petrukovich, and J.-A. Sauvaud, Statistical studies of geomagnetic storm dependencies on solar and interplanetary events: a review, Planet. Space Sci., 53(1-3), 189-196, 2005.

Yurchyshyn, V. B., H. Wang, P. R. Goode, and Y. Deng, Orientation of the magnetic fields in interplanetary flux ropes and solar filaments, Astrophys. J., 563, 381, 2001.

Zhang, J. et al., Solar and interplanetary sources of major geomagnetic storms (Dst<-100 nT) during 1996-2005, J. Geophys. Res., 112, A10102, doi:10.1029/2007JA012321, 2007.

Zhao, X. P. and D. F. Webb, Source regions and storm effectiveness of frontside full halo coronal mass ejections, J. Geophys. Res., 108(A6), 1234, doi:10.1029/2002JA009606, 2003.

N. Gopalswamy (e-mail: Nat.Gopalswamy@nasa.gov) 